\title{
Quantum Biology: A Scientific Revolution in our Understanding of Biological Systems
}

\section{Scott Waring}

Faculty of Science and Mathematics, Great Lakes College, Australia

*Corresponding author: Scott Waring, Faculty of Science and Mathematics, Great Lakes College, Australia, Tel: +61432069447; E-mail: scott.waring1@outlook.com

Received date: November 17, 2017; Accepted date: April 26, 2018; Published date: May 03, 2018

Copyright: (C) 2018 Waring S. This is an open-access article distributed under the terms of the Creative Commons Attribution License, which permits unrestricted use, distribution, and reproduction in any medium, provided the original author and source are credited.

\begin{abstract}
Until recently, the complexities of biological processes and systems have emphasized classifications and empirical rules, that have lacked explanatory power and been limited by many exceptions. On the other hand, modern physics and quantum physics in particular, has a universal capacity to explain physical interactions down to the subatomic scale, including biological processes and systems. The scientific field of biology has succeeded over the past decades in explaining macroscopic phenomena that are based on an improved understanding of molecular structures and behaviours. Likewise, quantum physics has provided a non-classical approach to unintuitive characteristics, and has recently been directed towards systems of increasing complexity. With the rise of high performance computing, the field of quantum biology has rapidly developed in recent years to challenge us to rethink biological processes such as the magnetoreception of Earth's magnetic field that allows birds to migrate, how enzymes are able to accelerate reactions at astonishing speeds, and why photosynthesis in plants is near to $100 \%$ efficient. Even biology's primary conceptual basis, namely Charles Darwin's and Alfred Wallace's theory of evolution by natural selection, is now thought to be influenced by the esoteric but fundamental laws of quantum mechanics. This article will act as a guide to explain such phenomena, explore the growing interconnectedness between the two scientific fields and question the future possibilities of quantum biology.
\end{abstract}

Keywords: Quantum; Biological systems; Sub-atomic; Photon; Photosynthesis; Enzyme; Evolution

\section{Introduction}

Why grass is green and blood is red: using quantum phenomena to explain biological systems.

This essay explores the emerging new field of quantum biology, in which biological systems can be explained at a fundamental level using quantum physics. In 1612, Donne wrote an elegy that was from the realm of abstract metaphysics in which he stated, 'Why grass is green, or why our blood is red, are mysteries which none have reached unto' [1]. He and others of that time period believed that there was no need for the inquisitive rational of science, or the natural curiosity that comes with being human. However much has changed in the last four hundred years. The interdisciplinary research from biologists and chemists has been able to explain that iron-based pigments with the ability to carry oxygen are at the center of haem chains in red blood cells [2]. Similarly, physicists also contributed to our understanding of the chlorophyll-containing mesophyll cells in plants. The colours expressed are explained with a combination of classical and modern physics. The energies absorbed and emitted, in both plant chlorophyll and blood haemoglobin molecules, by the quantum jumping of electrons, are the same as that which reflect green and red, respectively.

Quantum mechanics has long been known to play a crucial role in the world of chemistry and in turn, chemistry has been able to set the structured, predictable rules for biological systems (Figure 1). However, in the book 'What is Life?' Schrödinger questioned how an event at the atomic scale, a mutation in the molecular structure of a gene for example, whilst being governed by the laws of quantum mechanics, could cause a profound and visible change in the structure of a living organism [3].

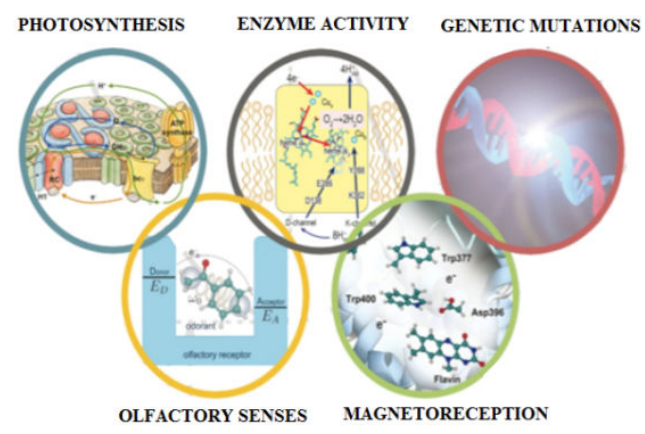

Figure 1: Quantum biology encompasses quantum mechanics to explain biological systems.

How can organisms undergo metamorphosis at such a rate that statistics and classical physics cannot explain? How is it possible that nearly all of photons energy can reach a leaf's reaction center without being reflected, absorbed or redirected? Seventy years since Schrödinger's book was published, scientific understanding has grown at an impressive and exponential rate; however questions like this are still difficult to approach. 


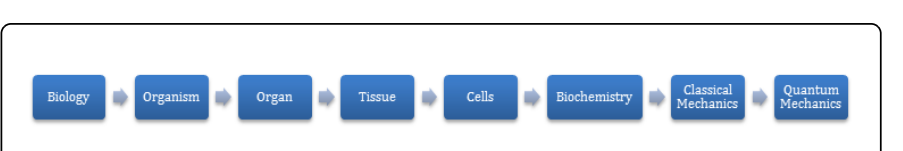

Figure 2: As lower levels are reached; atoms and subatomic particles are observed. Their relationships and behaviors are explained with quantum mechanics and lead to the many reactions seen in biological systems.

The effects of quantum mechanics can of course be observed, but usually only under extremely rigid conditions such as temperatures near absolute zero and under the influence of vacuums (Figure 2) [4]. Even then, researchers can only use simple particles on a very small scale to observe the complex effects of coherence [5]. Up until the late 1980 's, most scientists would agree that quantum mechanics played a trivial, if any, role in biological processes [6,7].

\section{Quantum Tunnelling}

\section{The Sun}

The energy provided from the Sun drives life on Earth, from metabolic reactions to reproducing behaviors. In order to supply this energy though, the Sun performs special types of reactions - fusion reactions. The sample hydrogen particles in the Sun fuse together to form helium and other heavier elements, releasing huge quantities of energy as a result. Essentially, the Sun is converting its mass into energy.

Since the 1920's, we have been researching and attempting to replicate the process of nuclear fusion, in order to harness the large amounts of energy for a variety of purposes. However, one of the main limitations on researching this nuclear process on Earth is achieving the required temperature needed for fusion to occur. The core of the Sun is estimated to be 15.6 million Kelvin and therein lays the problem. Researchers cannot effectively replicate these temperatures, so the search is focused on low-energy fusion reactions.

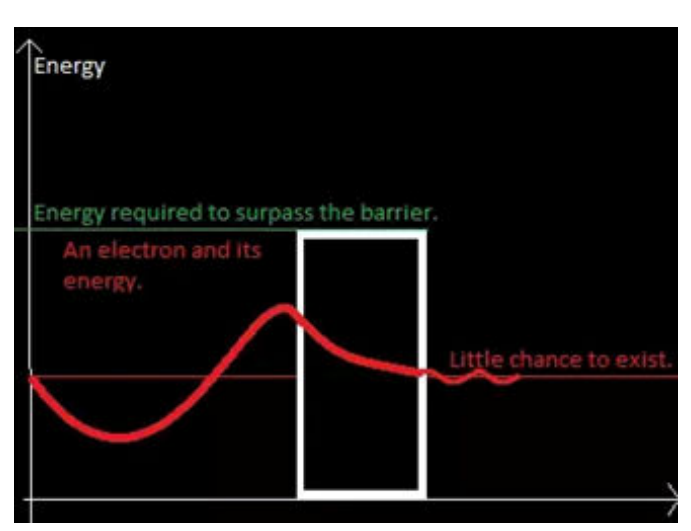

Figure 3: An electron does not have the required energy to get over the barrier to start a reaction. There is a small probability that it can tunnel through the barrier though and the reaction takes place as a result. This is how nuclear fusion works in the Sun, driving life on Earth [8].
As the hydrogen particles increase in temperature, there is a small chance that the hydrogen atoms will fuse together, even if the correct temperature has not been reached yet. This effect was mathematically introduced in 1928 by Hufbauer [9]. Balantekin and Takigawa demonstrated that rather than waiting for the required temperature needed to overcome the energy barrier that starts the fusion reaction, the electron in the hydrogen atom 'tunnels' through the barrier instead (Figure 3) [10].

The significance of this process is twofold. Firstly, our main source of heat and catalyst of many biological reactions relies on quantum mechanics to provide the sheer amount of energy required to drive biology processes on Earth. And secondly, if a structure as important and relatively simple as the Sun can use quantum forces, surely the incredibly complex and high number of processes that occur in biological systems, have undiscovered quantum connections.

\section{Enzyme catalysis}

The fastest studied reactions in the history of science include those in biological systems that are caused by proteins called enzymes. An appreciation of the sheer speed and rate of these reactions is yet to be completely realised by researchers but one thing is absolutely clear. In the absence of enzymes, these same reactions are among the slowest that have ever been measured, some with half-times approaching the age of the Earth [11]. After a substrate is bound at an enzyme's active site (forming an enzyme-substrate complex), its half-life is usually a small fraction of a second (Figure 4) [12].

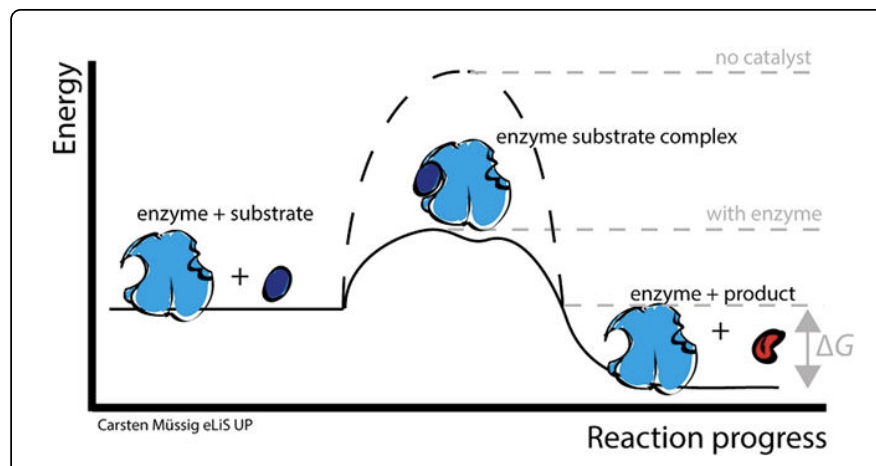

Figure 4: Lowering of reaction's activation energy caused by an enzyme-substrate complex. Also simulating a visual of the effects of quantum tunnelling on enzyme catalysis [13].

Back in 60 s, it was discovered that quantum tunnelling also takes place inside living cells [14]. Amphibians, such as frogs, use quantum tunnelling to undergo the biological process of metamorphosis where the tadpole breaks down and reassembles as a frog [15]. The speed at which this process occurs cannot be adequately explained by traditional methods of thinking. The tail is remarkably broken down into the basic units of life, and is redistributed in the newly forming frog as proteins, such as enzymes and collagen (a protein that supports the muscles, tendons and ligaments within living organisms and has elastic properties).

The strong bonds between proteins are very difficult to separate and should last much longer than the tadpoles lifespan [16,17]. Enzymes are responsible for breaking these bonds at an accelerated rate. The spontaneous rates of biological reactions examined in this way, span a 
range of more than sixteen orders of magnitude (Figure 5), with halflives ranging from five seconds, for the hydration of $\mathrm{CO}_{2}$ to 1.1 billion years, for the decarboxylation of amino acids $[18,19]$

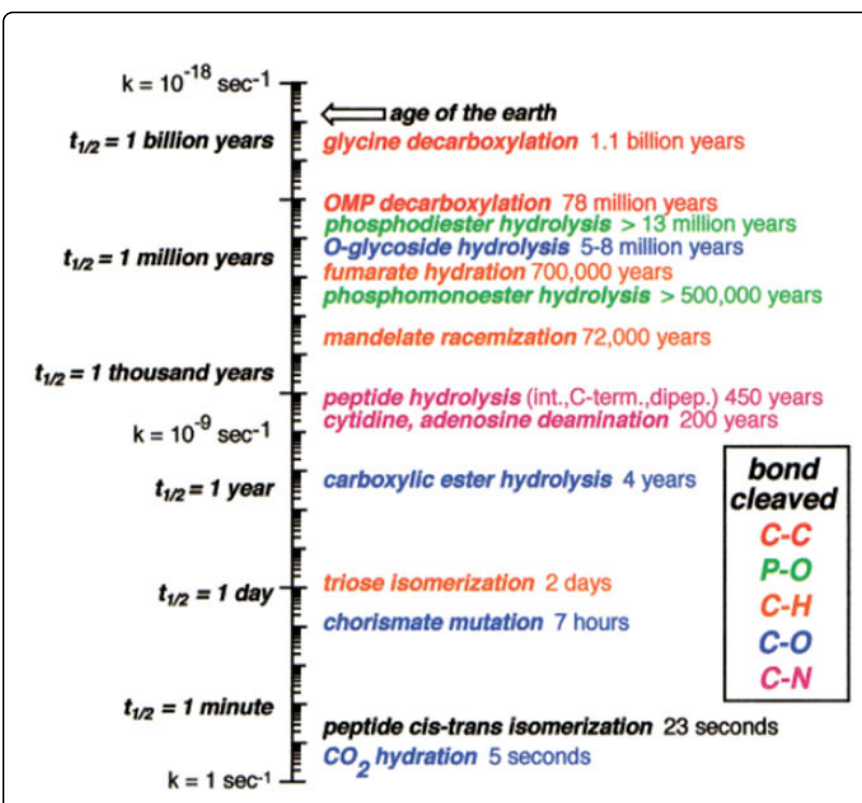

Figure 5: Natural half-times of some biological reactions in neutral solution at $25^{\circ} \mathrm{C}[20]$.

This incredible acceleration has been the subject of discussion and debate for nearly thirty years now and a leading theory has peaked the interests of physics and biologists alike. A group of scientists found that enzymes are able to transfer subatomic particles, like electrons and protons, from one part of a molecule to another, via quantum tunnelling [21]. It's an efficient theory and explains how the proton tunnelling to another location will cause the strong bonds to become weak bonds in a fraction of a second (Figure 6).

The protons do not have to overcome the energy barrier, but can tunnel through it (similar to tunnelling within the Sun), in order to break the chemical bonds. As subatomic particles are not solid, that can exhibit wave-like behaviours, allowing them to approach a barrier from one side and they have a certain probability of reappearing instantaneously on the other.

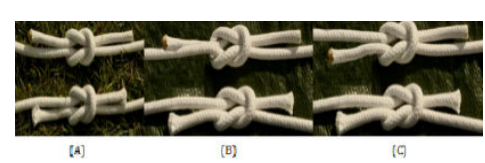

Figure 6: (A) Shows a simple but effective square knot at the top and the bottom shows a similar tie but will never join the two pieces of rope. This demonstrates the quantum effect of tunnelling in where, in this case, there are two possible outcomes-a strong bond and a weak bond. (B) Shows a digitally altered image where one of the ends of rope (the proton) on top is 'tunnelling' to a new position. (C) Illustrates the new position and now has effectively caused the bond to break. This could be demonstrated by a person pulling at any end on any rope in image (C). It would instantly come undone.
In metamorphosis, the collagen proteins in the tadpole tail are quickly broken down and other enzymes are then able to redistribute the particles to form the frog. However, some researchers are yet to be convinced that enzymes are doing anything special and that their own results suggest that "tunnelling is a fact of life, but life has no special effect on tunnelling" [22].

Contrasting such statements, with near overwhelming evidence, it is theorized that enzymes can use their dynamic behaviour to force protons through the energy barrier, a characteristic unique to these powerhouse proteins. Evidence is even beginning to emerge that such behaviours are selected by evolution to enhance enzyme catalysis [23]. Future research will provide intellectually provocative models of enzyme catalysis and suggest new avenues for experimental and computational investigations.

\section{Quantum Entanglement}

\section{Migration by magnetoreception}

The European robin (Erithacus rubecula) migrates from Scandinavia down to the Mediterranean, every autumn (Figure 7). Whilst migratory patterns with birds caused by seasonal changes is not a new phenomenon, what is special and worth investigating, is how they manage to travel to the exact same geographical areas each year. These areas are the same locations that their predecessors emigrated to and, hence, human curiosity has long wondered, how do organisms know exactly where they are going, particularly if they have never been there before? How can birds navigate with such accuracy? Is it a magnetic particle within them that responds to Earth's magnetic field? Is it a behavioural trait passed from one generation to the next? Or is it something smaller than a gene and much more complicated?

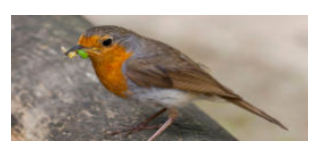

Figure 7: The European robin, and like a lot of other marine animals and even insects, navigate by sensing the Earth's magnetic field [24].

These hypotheses were experimentally tested in a repurposed planetarium (Figure 8); using specialised Emlen funnels which are round cages in the shape of funnels lined with scratch sensitive paper and an inkpad on the bottom, shown in Figure 9 [25].

At night, with the artificial magnetic field inactive, the caged migrant birds became restless and jumped more often in the direction where they intend to migrate, making ink prints on the scratch sensitive paper. However, what was even more astounding was that if the wavelength of light was changed, the behaviour of the robins changed and instead of moving in the direction of expected migration, their movements became random and they jumped in a variety of directions, as seen in Figure 10. It seemed that monochromatic blue light (the predominant wavelength of light at night) enhanced the robin's orienteering ability. 


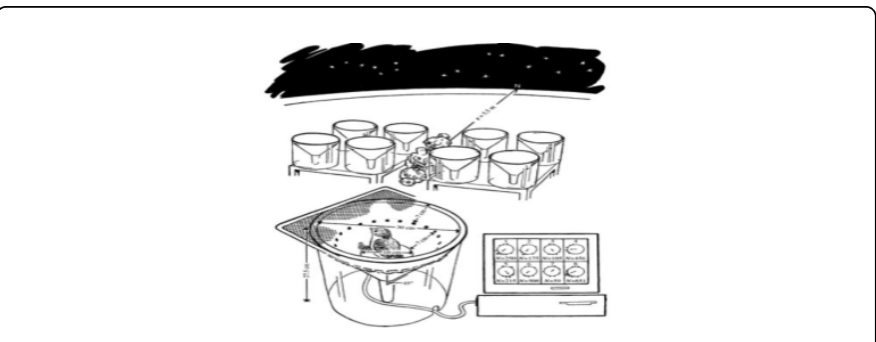

Figure 8: Sketch of the experimental equipment and set-up in the planetarium [26].

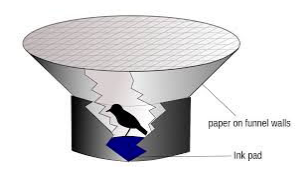

Figure 9: An Emlen funnel, used to document migration orientation in bird.

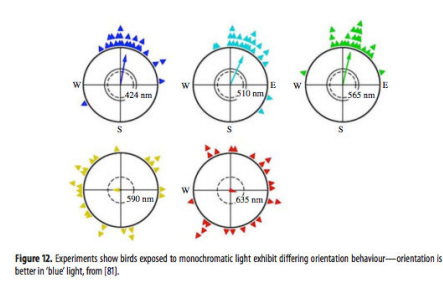

Figure 10: Experiments show birds exposed to monochromatic light exhibit differing orientating behaviour - orientation is better in 'blue' light [27].

The caged robins also changed their heading accordingly when the magnetic field was artificially altered using strong magnets, with variable directions being applied. This meant that when the magnetic field was active in a particular direction, the robin moved in the same direction. If living systems are sensitive to the magnetic vector potential component of the geomagnetic field, which is directed EastWest, this mean that they must have both $\mathrm{X}$ and $\mathrm{Y}$ coordinates available. This posed an interesting dilemma, was the underlying cause of this behaviour due to wavelengths of light or a varying magnetic field, like Earth's?

A researcher struggled for years to narrow down the possible reasons that would explain such behaviour. Eventually he developed leather hoods (Figure 11) with removable eye patches which were able to cover one eye of the robin at a time during the experiments. It was found that photons excited a particular protein molecule, cryptochrome, in the retinal cells of the right eye and causing an electron from a particular particle to move to another. This means both molecules are now charged, that is to say one molecule is positive and one is negative[28]. The incoherent part of this anomalyis that these molecules are both positive and negative at the same time. They exhibit behaviours of both charges until one is measured, and, hence, the other particle when measured will be the opposite charge, due to the Pauli exclusion principle [29]. These particles are now in a state of quantum entanglement where they are correlated with each other, but they lose their individuality [30].

The infamous example of Schrodinger's Cat helps explain this unusual concept. If you were to place a cat into a box containing a randomly-released lethal gas and sealed the box up, the cat itself could be thought of as both dead and alive, by an external observer [31]. The charged particles mentioned above, exhibit such behaviours and hence can be thought of in the same way as Schrodinger's Cat.

Entangled particles are extremely sensitive to magnetic fields. Their interactions, caused by the angle of inclination of the magnetic field, trigger action potentials and the subsequent chemical reactions act as sensory chemicals which tell the robin which way to go [31]. The entangled pair induces different chemical reactions based on their relationship and quantum state (orientation of spin). The tiny variations in Earth's magnetic field can alter the state of these entangled electrons and hence affect the robin's ability to sense its orientation. Even Einstein hated this idea; he called it "spooky action at a distance" as entanglement implied that the relationships between particles occurred faster than the speed of light, impossibility in Einstein's theory of relatively.

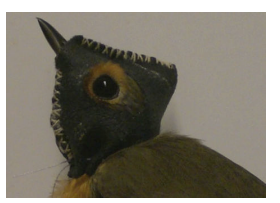

Figure 11: The leather hood used to cover the right eye of a European robin - the source of cryptochrome and the inclination compass used for migratory orientation [32].

The implications of research could be ground breaking. Many organisms use cryptochrome as a photoreceptor so this quantumassisted magnetic sensing, could be widespread. Not only birds, but also some insects and even plants show physiological responses to magnetic fields. An example includes the growth-inhibiting influence of blue light on the flowering plant Arabidopsis thaliana and it is moderated by magnetic fields [33].

\section{Quantum Coherence}

\section{Photosynthesis}

In the cases of the avian compass described above, the primary evidence for quantum processes is observation of animal behaviour, combined with a lack of plausible classical models. However, by contrast, evidence for quantum influences in the biological process of photosynthesis is direct, measurable and overwhelming.

Quantum coherence is the idea of quantum entities multitasking. It can be represented by the quantum skier from Figure 12 where an object behaves like a wave so it doesn't just move in one direction or the other, but can follow multiple pathways at the same time. 
Page 5 of 8

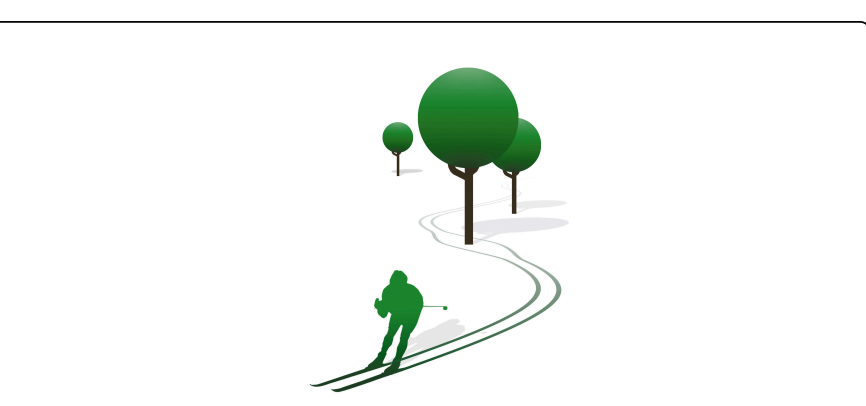

Figure 12: The quantum skier counter intuitively exhibits a behaviour of being able to follow several paths at once and 'finds' the most efficient one to perform its designated process [34] .

A light harvesting, photosynthetic system uses 'antenna' molecules called chlorophyll inside chloroplasts, to capture photons, the quantum of light (sunlight energy). This capturing of photons knocks out an electron from the chlorophyll molecule and creating a quantum particle called an exciton.

The exciton travels through surrounding chlorophyll molecules, until it reaches the reaction centre and ultimately results in the production of Adenosine trisphosphate (ATP), the 'molecule of life' via aerobic cellular respiration.

For maximum efficiency, all photons absorbed have to make it to the reaction centre, but there are so many random paths and the photon does not 'know' the most efficient route [35]. If the exciton doesn't reach the reaction centre fast enough, the energy will dissipate into the system. So how then can the process of light-harvesting, by nearly $100 \%$ efficient?

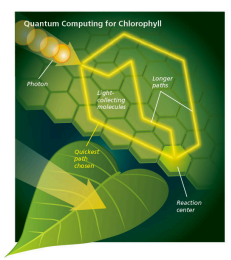

Figure 13: The coherence of quantum particles allows the wave-like properties to be examined and the most efficient path of the photon energy (via the exciton) will be the result [40].

The evidence from multiple, independent studies, is that rather than taking one path, quantum coherence is used to travel all paths simultaneously as shown in Figure 13 [36-39].

Therefore, there is plenty of evidence that can demonstrate that the interplay between quantum coherence and the performance of biomolecular complexes. This can be intuitively explained by the fact that quantum coherence allows the system to explore several network paths in parallel to achieve the desired task to find the optimal solution and leave out inefficient slower pathways [41].

\section{Quantum Biology: The Future}

\section{The theory of evolution - Quantum edition}

The final question that this paper will explore is whether quantum tunnelling plays a non-trivial role in mutations in DNA. It has been well established that variations (mutations) within species drives evolution [42].

DNA is comprised of complementary nitrogenous bases (adenine and thymine, cytosine and guanine) and during the cell cycle, the majority of time is spent in a stage called interphase [43]. This is where the cell performs its normal functions including the process of DNA replication. Essentially, this is how one strand of DNA is replicated to form 2 identical strands for the purpose of cell division (mitosis or meiosis).

The two strands of DNA found in a double-helix structure, are held together by these nitrogenous bases; like a twisted ladder [44]. These bases are bound together by relatively weak hydrogen bonds (protons). The process of DNA replication is always proofed and checked for errors by an enzyme named DNA polymerase which can repair most mutations; however polymerase still has its limitations [45]. Sometimes in the process, mistakes can occur and these mistakes are called genetic mutations.

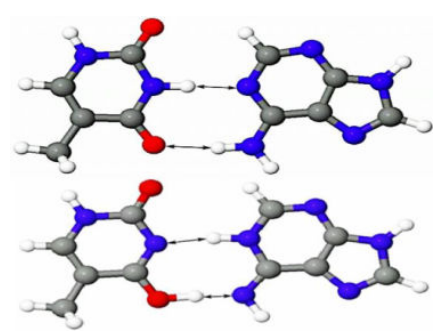

Figure 14: The white atoms are hydrogen and protons from the hydrogen atoms can "jump" across to the other side via quantum tunnelling. If the strands of DNA are separated by DNA replication or protein synthesis, while the hydrogen has jumped across to the opposite side, these strands may not replicate or transcribe correctly-this is a mutation [46].

This movement can be explained by the process of quantum tunnelling [47]. When replication is initiated by an enzyme called DNA helicase, which splits hydrogen bonds [48]. The two white balls in the middle of Figure 14 are protons, one proton prefers to sit on one strand; the other, on the other strand.

They have ability to tunnel through the bonds to the other side. If the two strands of DNA then separate for replication to occur, and the two protons are in the wrong positions, this could ultimately lead to a mistake known as a mutation(Figure 15). 


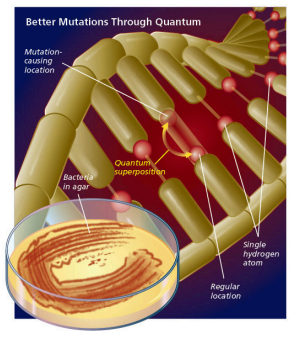

Figure 15: DNA mutates when this hydrogen atom tunnels, quantum-mechanically, to the "wrong" half of its rung. The property of coherence, before it is observed, the atom will simultaneously exist in both a mutated and non-mutated state-that is, it would sit on both sides of the rung at the same time [49].

This concept has been examined using bacteria. A population of bacteria (E. coli) with hydrogen nuclei was cultured and the mutations in the produced cells were quantified. At the same time, a separate population was cultured, but these had deuterium nuclei (deuterons), a heavier isotope of hydrogen [50]. The results of these experiments showed that the protons within hydrogen nuclei were able to tunnel easily and thus led to many mutations. However, the population containing deuterons did not produce many mutations. This is because deuterons are twice as heavy as the hydrogen nuclei and therefore are increasingly difficult to tunnel through energy barriers.

The concept of quantum mechanics playing a non-trivial role in DNA mutations is still heavily debated today, predominated because the concentrated deuterium oxide will not take a frequency imprint until about $4 \%$ of hydrogen oxide is added. This suggests that there are 'windows' of opportunity in which biological systems are sensitive to the electro quantum environment, leading to a possible eventuality of changes in the genetic information carried by individual organisms. Current research certainly allows for quantum tunnelling to be a plausible mechanism for variations to occur and hence have the opportunity to be naturally selected to survive in certain conditions. It's even speculated that through the process of quantum coherence, living cells act as biological quantum computers and are able to explore multiple possible mutational states simultaneously, and select those states that provide the greatest advantage - The Theory of Evolution redefined as a construct of quantum mechanics.

\section{Approaching an era of quantum technologies}

Quantum coherence or superposition is an extremely efficient energy transportation system. The photosynthesis example discussed above demonstrated that particles can travel faster along the photosynthetic complex due to the fact that it can exist in two states simultaneously. Researchers from the PAPET's group shot fast laser pulses into biological systems and observed interference along the transport network, a signature of wavelike phenomena. As a result, we are able to obtain a more fundamental understanding of how photosynthesis works. And can guide future research towards designing much more efficient solar cells than the ones we currently have [50]. However, they do outline that one of the biggest obstacles in designing complex organic solar cells, is the difficulty to control what happens after light is absorbed. The characteristics that would be most valuable and beneficial include the transmission of energy, storing information or emitting light [51].
An extended application of solar-cells is the development of bioinspired sensors. Developing sensors that interpret desired signals whilst ignoring complex background influences, as well as lightweight, affordable and retain their sensitivity is particularly difficult [52]. Nature is an extraordinary test bed for biological systems as they've been exposed to billions of years of evolution and can efficiently use quantum mechanics to their advantage. Exploring magnetic navigation, Quantum Effects in Biological Environments (QuBE) researchers from the Defence Advanced Research Projects Agency (DARPA) are also studying photosynthesis, olfaction and the underlying theoretical framework needed to link biology and quantum phenomena [53].

Another area of potential application is in quantum computing [54]. Quantum data (qubits) can exist in both states at once, thus permitting the simultaneous exploration of all possible answers to the computation that they encode, similar to the quantum coherence within a photosystem, performing a 'best-path' quantum computation, or the mutated state of DNA. It has been shown through studying how water can embed or imprint frequencies that the 3 basic logic gates needed for biological quantum computers to operate, can be assembled and maintained in water. Even with such advancements, research still goes on to suggest that these gates are so sensitive that they can be detected and measured using nerve impulses. Quantum simulation has potential applications for the study of condensed matter physics, material science, and high-energy physics [55-57].

\section{Conclusion}

If the question is put forth, do quantum mechanics play a significant role in biological systems? The answer is most definitely yes. Our Sun employs quantum tunnelling to transform hydrogen to helium which in turn releasing heat and light energy. This effect alone allowed Earth able to become habitable. The evolution of photosynthetic organisms utilized the Sun's energy and transformed the Earth from anoxic to oxic. Every organism, since this epic event in our history has evolved as a direct result of quantum influences.

The design of experiments that directly test hypotheses here, at the quantum level, must be a priority for advancement. Much of quantum biology remains as conjecture because of the amount of restrictions and environmental controls needed to observe even the simplest quantum effect. Any analysis of such data in relation to biological systems is redundant without experimental proof from complex living organisms. At this stage, there is too much conflict within data to suggest quantum mechanics can explain all biological systems. However, there is enough evidence to suggest an undeniable plausibility. The exploration of quantum biology certainly has many uses in various fields, and is definitely an area of research that will expand.

The future on these biological processes is an exciting one, providing intellectually provocative, biologically centric models that suggest new avenues for experimental and computational investigation in a 'quantum leap' effort to discover if evolution, Darwin's and Wallace's (and the scientific field of Biology) keystone theory, is responsible for the incoherent mechanisms that drive biological processes, and indeed, life itself. Even Schrodinger [3] himself implied that the behaviour of living organisms approaches quantum mechanical behaviours and that all biological systems involve the movement of fundamental, quantum particles within their cells as described by quantum mechanics. 


\section{References}

1. Donne J (1886) The Poems of John Donne. London: Lawrence \& Bullen.

2. BreathnachCS (2014) Claude Bernard and his revelations in physiology. Ir J Med Sci 183: 139-146.

3. Schrodinger E (1944) What is life? The physical aspect of the living cell. London: Cambridge University Press.

4. Hackermuller L, Hornberger K, Brezger B, Zeilinger A, Arndt M (2004) Decoherence of matter waves by thermal emission of radiation. Nature 427: 711-714.

5. Hackermuller L, Uttenthaler S, Hornberger K, ReigerE, Brezger B, et al. (2003) Wave nature of biomolecules and fluorofullerenes. Phys Rev Lett 91: 090408

6. Ball P (2015) Biology: An introduction. The Royal Institute.

7. Longuet-Higgins HC (1962) Quantum mechanics and biology. Biophys J 2: 207-215.

8. Quora (2016) What causes quantum tunnelling?

9. Hufbauer K (2009) National Academy of Sciences.

10. Balantekin A, Takigawa N (1998) Quantum tunneling in nuclear fusion. Rev Mod Phys 70: 77-100.

11. Garcia-Viloca M, Gao J, Karplus M, Truhlar DG (2004) How enzymes work: Analysis by modern rate theory and computer simulations. Science 303: 186-195.

12. Frick L, Neela JP, Wolfenden R (1987) Transition state stabilization by deaminases: Rates of nonenzymatic hydrolysis of adenosine and cytidine. Bioorg Chem 15: 100-108.

13. Dictionary B (2017) Enzyme-substrate complex.

14. De Vault D, Chance B (1966) Studies of photosynthesis using a pulsed laser: I. Temperature dependence of cytochrome oxidation rate in chromatium: Evidence for tunneling. Biophys J 6: 825-847.

15. Gross J, Lapiere CM (1962) Collagenolytic activity in amphibian tissues: A tissue culture assay. Proc Natl Acad Sci U S A 48: 1014-1022.

16. Wu C, Ito K, Buytendyk A, Bowen K, Wu J (2017) Enormous hydrogen bond strength enhancement through $\pi$-conjugation gain: Implications for enzyme catalysis. Biochemistry 56: 4318-4322.

17. Roughton F (1941) The kinetics and rapid thermochemistry of carbonic acid. J Am Chem Soc 63: 2930-2934.

18. Snider MJ, Wolfenden R (2000) The rate of spontaneous decarboxylation of amino acids. J Am Chem Soc 122: 11507-11508.

19. Wolfenden R, SniderMJ (2001) The depth of chemical time and the power of enzymes as catalysts. Acc Chem Res 34: 938-945.

20. Cha Y, Murray CJ, Klinman JP (1989) Hydrogen tunneling in enzyme reactions. Science 243: 1325-1330.

21. Doll KM, Finke RG (2003) A compelling experimental test of the hypothesis that enzymes have evolved to enhance quantum mechanical tunneling in hydrogen transfer reactions: The $\beta$-neopentylcobalamin system combined with prior adocobalamin data. Inorg Chem 42: 4849-4856.

22. Delgado M, Gorlich S, Longbotham JE, ScruttonNS, Hay S, et al. (2017) Convergence of theory and experiment on the role of preorganization, quantum tunneling and enzyme motions into flavoenzyme-catalyzed hydride transfer. ACS Catalysis 7: 3190-3198.

23. Klinman JP, Kohen A (2014) Evolutionary aspects of enzyme dynamics. J Biol Chem 289: 30205-30212.

24. Klinman JP, Amnon K (2013) Hydrogen tunneling links protein dynamics to enzyme catalysis. Klinman, J.P. \& Amnon, K. Hydrogen Tunneling Links Protein Dynamics to Enzyme Catalysis. Annu Rev Biochem 82: 471-496.

25. DARPA (2014) Study on magnetic compass orientation in birds builds case for bio-inspired sensors.

26. Emlen ST, Emlen JT (1966) A technique for recording migratory orientation of captive birds. The Auk: Ornithological Advances 83: 361-367.
27. Mouritsen H, Larsen ON (2001) Migrating songbirds tested in computercontrolled Emlen funnels use stellar cues for a time-independent compass. J ExpBiol 204: 3855-3865.

28. Wiltschko R, Wiltschko W (2007) Magnetorecption in birds: Two receptors for two different tasks. J Ornithol 148: 61-76.

29. Pauli W (1947) Exclusion principle and quantum mechanics. Neuchâtel: Editions du Griffon.

30. Morello A (2014) Bird navigation: The quantum around you. Sydney, NSW, Australia: University of New South Wales: elearning .

31. Schrodinger ER (1935) Die gegenwärtige Situation in der Quantenmechanik. Nat Sci 23: 807-812.

32. Ritz T, Adem S, Shulten K (2000) A model for photoreceptor-based magnetoreception in birds. Biophys J 78: 707-718.

33. Engels S, Hein C, Lefeldt N, Prior H, Mouritsen H (2012) Nightmigratory songbirds possess a magnetic compass in both eyes. PLoS One 7: 1-8.

34. Ooi A, Wong A, Ng TK, Marondedze C, Gehring C, et al. (2016) Growth and development of Arabidopsis thaliana under single-wavelength red and blue laser light. Sci Rep 6: 1-13.

35. Al-Khalili J (2011) Just when you think you understand quantum physics. Surrey Physics Blog.

36. Romero E, Augulis R, Novoderezhkin V, Ferretti M, et al. (2014) Quantum coherence in photosynthesis for efficient solar-energy conversion. Nat Phys 10: 676-682.

37. O'Reilly EJ, Olaya-Castro A (2014) Non-classicality of the molecular vibrations assisting exciton energy transfer at room temperature. Nat Commun 5: 1-10.

38. Collin E, Wong C, Wilk K, Curni P, Brumer P, et al. (2010) Coherently wired light-harvesting in photosynthetic marine algae at ambient temperature. Nature 463: 644-647.

39. Ball P (2011) Physics of life: The dawn of quantum biology. Nature 474: 272-274.

40. Fleming GR, Engel GS, Calhoun TR, Read EL, Ahn T, et al. (2007) Evidence for wavelike energy transfer through quantum coherence in photosynthetic systems. Nature 446: 782-786.

41. Merali Z (2014) Solving biology's mysteries using quantum mechanics.

42. Caruso F (2016) What is quantum biology? Lindau Nobel Laureate Meetings.

43. Tarlach G (2014) Mutation, not natural selection, drives evolution. Discover, pp: 12-17.

44. Murray AW, Kirschner MW (1989) Dominoes and clocks: the union of two views of the cell cycle. Science 246: 614-621.

45. Watson JD, Crick FH (1953) A structure for deoxyribose nucleic acid. Nature 171: 737-738.

46. Saiki RK, Gelfand DH, Stoffel S, Scarf SJ, Hiquchi R, et al. (1988) Primerdirected enzymatic amplification of DNA with a thermostable DNA polymerase. Science 239: 487-491.

47. Wellford S (2016) Quantum biology.

48. Lowdin PO (1963) Proton tunneling in DNA and its biological implications. Rev Mod Phys 35: 724-732.

49. Schaeffer L, Richard R, Humbert S, MoncollinV, Vermeulen W, et al. (1993) DNA repair helicase: A component of BTF2 (TFIIH) basic transcription factor. Science 260: 58-63.

50. McFadden J, Al-Khalili J (1999) A quantum mechanical model of adaptive motion. Biosystems 50: 203-211.

51. Physics Organisation (2015) Quantum physics provides startling insights into biological processes.

52. Mirkovic T, Ostroumov E, Anna J, Grondelle R, Scholes G (2016) Light absorption and energy transfer in the antenna complexes of photosynthetic organisms. Chem Rev 117: 249-293.

53. Wolf P (2017) Bio-inspired sensors. APS Physics.

54. DARPA (2014) Study on magnetic compass orientation in birds builds case for bio-inspired sensors. 
Citation: Waring S (2018) Quantum Biology: A Scientific Revolution in our Understanding of Biological Systems. Biol Syst Open Access 7: 185. doi:10.4172/2329-6577.1000185

Page 8 of 8

55. Balantekin A, Takigawa N (1998) Quantum tunneling in nuclear fusion. Rev Mod Phys 70: 77-100.

56. Cai JM (2017) Quantum simulation meets quantum biology. Science China 60: 1-2.
57. Georgescu IM, Ashab S, Nori F (2014) Quantum simulation. Rev Mod Phys 86: 152-193. 